\title{
Corneal thickness changes following cataract surgery: effect of lens implantation and sodium hyaluronate
}

\author{
J S H JACOB \\ From the West of England Eye Infirmary, Exeter
}

\begin{abstract}
SUMMARY Consecutive patients undergoing intracapsular cataract surgery with and without lens implantation were included in one of three groups of fifteen patients for postoperative corneal thickness measurement. One group underwent cataract extraction alone, another group had cataract extraction and lens implantation using iris supported posterior chamber implants, and another group underwent lens implantation with the use of sodium hyaluronate to reform the anterior chamber after cataract extraction. Maximal increase in corneal thickness occurred within the first 24 hours of surgery in all groups. No significant difference in corneal thickness between the implant and non-implant group was found at any time. When sodium hyaluronate was used to reform the anterior chamber prior to lens implantation the increase in corneal thickness was significantly less than in the group not on sodium hyaluronate $(0.01<p<0.02$ on the third postoperative day). In the long term all patients' corneas returned to preoperative levels within 28 days of surgery.
\end{abstract}

Every cataract extraction even by today's microsurgical techniques involves some corneal manipulation and endothelial damage. Severe damage leads to late corneal decompensation, which has been reported as occurring in $1 \%$ of cataract extractions.' Interest has therefore developed in the assessment of endothelial damage either by cell counts or indirectly by measuring corneal thickness postoperatively. Miller and Dohlmann ${ }^{2}$ recorded a significant increase in corneal thickness as long as six months after cataract extraction performed by a Graefe section. A $15 \%$ increase on the second day, with a $9 \%$ increase on the sixth postoperative day, was reported by Norn. ${ }^{3}$ An $11.6 \%$ increase at four days with a return to normal within two weeks was reported by Wood and Maumanee. ${ }^{4}$ Lens implantation was shown ${ }^{5}$ further to increase corneal thickness in a series using Binkhorst iris fixated anterior chamber lenses. The average corneal thickness in this series increased during the third to fifth postoperative day to $21 \cdot 7 \%$. Similarly, Cheung et al. ${ }^{\circ}$ found a significantly greater increase in corneal thickness on the fifth postoperative day in eyes with Federov or Binkhorst implant than in non-implanted eyes. A good correlation between endothelial cell loss measured by specular

Correspondence to Mr J S H Jacob, FRACS, West of England Eye Infirmary, Magdalen Street, Exeter EX2 4HT. microscopy and early postoperative increase in corneal thickness was found in these patients. Miller and Stegman, using sodium hyaluronate to protect the endothelium prior to lens implantation, found a reduction in increase of corneal thickness.?

This study was undertaken to assess three aspects: (1) to measure the very early changes of corneal thickness following cataract extraction and cataract extraction plus lens implantation; (2) to compare thickness changes in a group without implant with those in a group of patients implanted with the modern generation of iris supported postchamber lens; (3) to observe the effect on corneal thickness of sodium hyaluronate used in conjunction with lens implantation.

\section{Patients and methods}

Forty-five consecutive patients who satisfied the exclusion criteria and were attending the West of England Eye Infirmary for cataract surgery were included in the series. They were subdivided into three groups of 15 patients. One group underwent cataract extraction alone, one group had cataract extraction plus lens implantation, and a third group in which sodium hyaluronate was used in conjunction with lens implantation. 
EXCLUSION CRITERIA

Any patient with endothelial defect, either dystrophy or cornea guttata, or any pre-existing ocular disease other than cataract was excluded. Cases were removed from the trial if there were operative complications such as vitreous loss or endothelial contact with an implant. Patients who developed complications postoperatively such as a shallow anterior chamber with wound leakage or implant dislocation were also removed from the series.

\section{OPERATIVE TECHNIQUE}

Lens extraction was performed under general anaesthesia by microsurgical techniques. All but three cases were operated on by the author. A posterior corneal section of approximately $120^{\circ}$ was made with either a diamond knife or razor fragment. The cornea was retracted by the assistant using Colibri forceps to grasp the upper cut edge of the corneal wound and the lens extracted with a cryoprobe without the use of alpha chymotrypsinogen. The cornea was released and the anterior chamber reformed with air. In the implant group the intraocular lens, either Little Arnott, Boberg-Ans, or Severin Lens, was inserted under the air bubble through the wound with minimal retraction of the wound edge. Implantation into the pupil was achieved by a one-movement technique, engaging the iris immediately with the lower loops, pushing down until the other loops became engaged in the iris. In the group on sodium hyaluronate this material was used to reform the anterior chamber prior to lens implantation. A peripheral iridectomy was performed in all cases and the incision closed with a continuous $10 / 0$ monofilament nylon suture. All the remaining air was replaced by balanced salt solution at the end of the procedure. No attempt was made to remove the sodium hyaluronate from the anterior chamber in the group on sodium hyaluronate. Chloramphenicol and pilocarpine $4 \%$ drops were instilled at the end of the operation on the table.

\section{POSTOPERATIVE REGIMEN}

All patients were maintained on pilocarpine drops $2 \%$ twice daily and prednisolone drops $0.5 \%$ with neomycin $0.5 \%$ four times daily for two weeks, the dosage reducing to twice daily for one month before it was stopped.

\section{MEASUREMENT OF CORNEAL THICKNESS}

Central corneal thickness was measured with a HaagStreit pachometer which was modified according to Mishima and Hedbys ${ }^{8}$ to ensure accurate measurement of the same area of the cornea. The modifications (Fig. 1) were made by the University of Exeter Department of Engineering and consist of extending

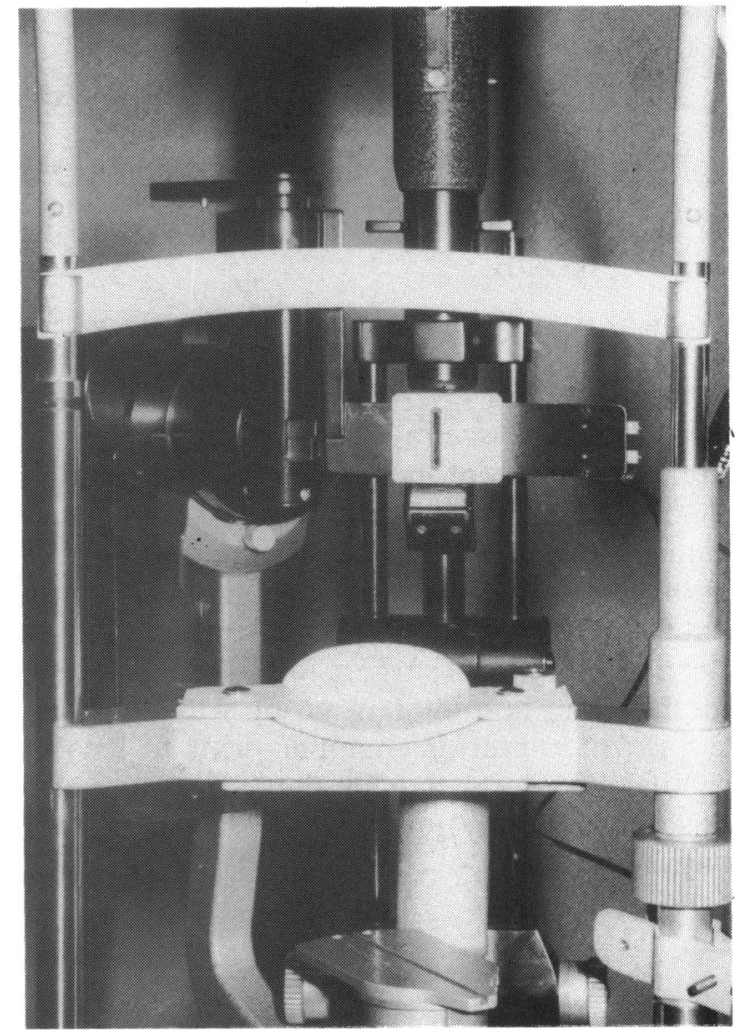

Fig. 1 Modified Haag-Streit pachometer attached to slitlamp.

the metal diaphragm of the Haag-Streit pachometer by approximately twice the length of the original. At an equal distance from the rotating prism axis two $1 \mathrm{~mm}$ fibre optic light ends were inserted $5 \mathrm{~mm}$ above and below the centre of the extension. The light source for the fibre optic cable was supplied from a $12 \mathrm{~V}$ car bulb.

After the slit-lamp is adjusted for height the light beam is perpendicular to the cornea, with the patient looking straight at the slit beam when the two small lights are reflected from the corneal epithelium along the same line above and below the horizontal. The central corneal thickness is then obtained by rotating the sector shaped disc on the pachometer in the usual manner until the endothelium of the upper field is in line with the epithelium of the lower, the images of the two pinpoint lights reflected from the epithelium being kept equidistant from the horizontal line. The scale reading on the sector gives the central corneal thickness in $\mathrm{mm}$. Preoperative measurements were taken on the day prior to surgery and then following surgery at 4 hours, 8 hours, 24 hours, and then every 12 hours for a total of 96 hours, when the patients were discharged from hospital. Late measurements 

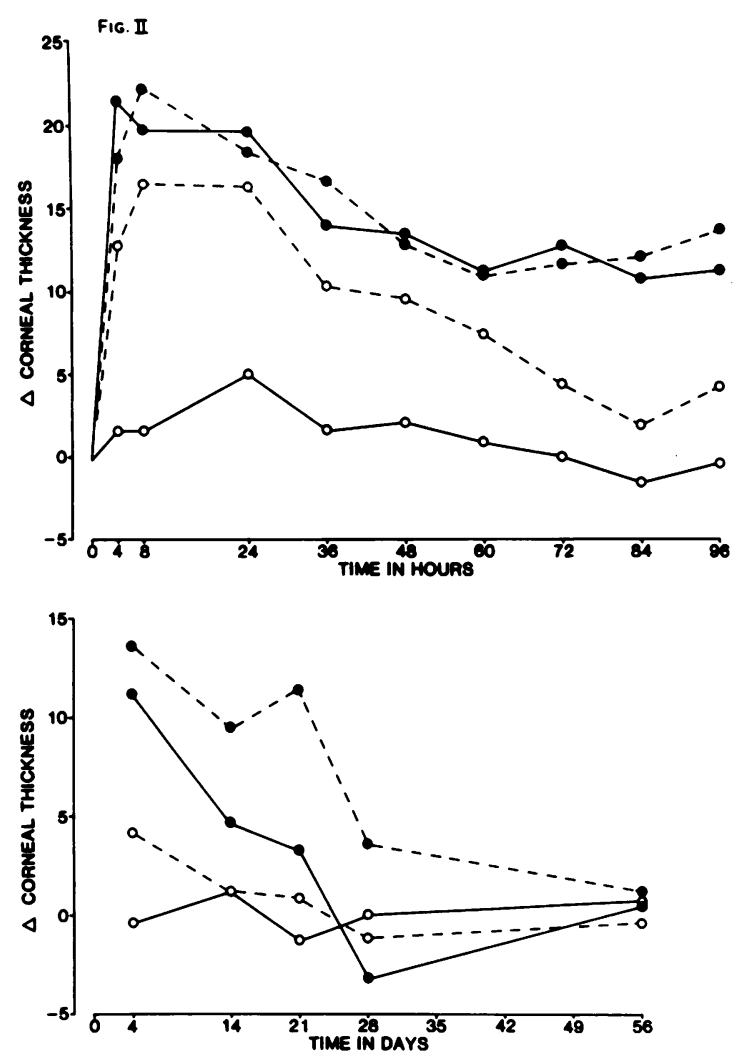

Fig. 2 Solid circles with broken line represent the cataract extraction group plus lens implantation without sodium hyaluronate. Solid circles joined by continuous line represent plain cataract extraction without sodium hyaluronate. Open circles joined by broken line represent cataract extraction plus lens implantation with sodium hyaluronate. Open circles joined by continuous line represent control group without surgery.

were made at two weeks, three weeks, four weeks, and eight weeks postoperatively for all groups. The unoperated eye was similarly measured in 15 cases to act as control.

\section{Results}

The early and late mean percentage changes in corneal thickness are shown graphically in Fig. 2 for all groups of patients and controls. In all groups there was a return to preoperative thickness by the end of 28 days.

Plain lens extraction versus lens extraction plus lens implantation. Increase in corneal thickness in these two groups is shown in Table 1. There was no statistically significant difference between these two groups by Student's $t$ test. The maximum increase in corneal thickness in both groups was seen in the first
24 hours, at 4 hours for the plain lens extraction and at 8 hours for the lens extraction with implantation. Return to preoperative thickness was slightly quicker in the plain extraction group, with only a $4.76 \%$ increase at two weeks compared with $9.5 \%$ for extraction plus implantation, though this was not statistically significant.

Lens extraction plus implantation versus lens extraction plus implantation with use of sodium hyaluronate. The increase in corneal thickness in this group is shown in Table 2. Although the mean corneal thickness was consistently less in the sodium hyaluronate group than in the group not on sodium hyaluronate group (Fig. 2), it became statistically significant only at 84 hours $(0.01<p<0.02)$.

\section{Discussion}

Comparisons between corneal thickness and endothelial damage by means of specular microscopy have in the past shown a good correlation between the increased cell loss and early postoperative increase in corneal thickness. ${ }^{69}$ Measurement of corneal thickness is a more readily available method and may in some ways be a better overall indicator of endothelial cell damage than counting endothelial cells over a very small area of the cornea. In this series damage to the endothelium resulted in maximal corneal oedema at 4 hours postoperatively in the plain extraction, at 8 hours postoperatively in extraction with implantation, and at 24 hours postoperatively with the use of sodium hyaluronate for endothelial protection. A peak in corneal thickness at 24 hours has been noted previously, but measurements prior to 24 hours have not been recorded by other workers. The peak increase within 24 hours may account for the clinical appearance of slight shallowing of the anterior chamber on the second and third postoperative days, when a reduction in corneal oedema leads to slight wound leakage. The maximum increase in corneal thickness of between $22.2 \%$ and $16.5 \%$ within 24 hours may be compared with other known recent series $-21.7 \%^{5}$ at third to fifth day ${ }^{5}$ and $27.8 \%$ at 24 hours. $^{6}$

In the present series no statistical significance in corneal thickness was found between the implant and non-implant groups. However, previous series have shown differences between lens implant and nonimplant groups. But the present series is different from the others in that the newer generation of posterior chamber iris-supported lenses are used and cases with endothelial contact were excluded. It indicates that with modern techniques and careful surgery endothelial damage can be minimised to approach that of plain lens extraction.

In Miller and Dohlmann's series ${ }^{2} 76 \%$ of the 
Table 1 Mean corneal thickness measurements $(\mu \mathrm{m})$ for plain cataract extraction and extraction plus implantation

\begin{tabular}{|c|c|c|c|c|c|c|c|c|c|c|}
\hline Time (h) & Preop. & 4 & 8 & 24 & 36 & 48 & 60 & 72 & 84 & 96 \\
\hline Plain extraction & 479 & 581 & 573 & 577 & 576 & 543 & 530 & 540 & 530 & 536 \\
\hline \multirow[t]{2}{*}{$\begin{array}{l}\text { Extraction }+ \\
\text { implant }\end{array}$} & 454 & 535 & 554 & 537 & 529 & 512 & 504 & 504 & 506 & 590 \\
\hline & & \multicolumn{2}{|c|}{2 Weeks } & & \multicolumn{2}{|l|}{4 Weeks } & \multicolumn{2}{|c|}{8 Weeks } & & \\
\hline \multicolumn{2}{|l|}{ Plain extraction } & \multicolumn{2}{|c|}{501} & & \multicolumn{2}{|l|}{494} & \multicolumn{2}{|c|}{482} & & \\
\hline \multicolumn{2}{|l|}{$\begin{array}{l}\text { Extraction }+ \\
\text { implant }\end{array}$} & \multicolumn{2}{|c|}{497} & & \multicolumn{2}{|l|}{470} & \multicolumn{2}{|l|}{459} & & \\
\hline
\end{tabular}

Table 2 Mean corneal thickness measurements $(\mu \mathrm{m})$ for cataract extraction plus implantation and extraction with lens implant under sodium hyaluronate

\begin{tabular}{|c|c|c|c|c|c|c|c|c|c|c|}
\hline Time (h) & Preop. & 4 & 8 & 24 & 36 & 48 & 60 & 72 & 84 & 96 \\
\hline $\begin{array}{l}\text { Extraction }+ \\
\text { implant }\end{array}$ & 454 & 535 & 554 & 537 & 512 & 504 & 504 & 504 & 506 & 509 \\
\hline \multirow{2}{*}{$\begin{array}{l}\text { Extraction }+ \\
\text { implant }+\mathrm{Na} \\
\text { hyaluronate }\end{array}$} & 474 & 534 & 532 & 551 & 523 & 513 & 590 & 495 & 483 & 494 \\
\hline & & \multicolumn{2}{|c|}{2 Weeks } & & \multicolumn{2}{|l|}{4 Weeks } & \multicolumn{2}{|c|}{8 Weeks } & & \\
\hline $\begin{array}{l}\text { Extraction+ } \\
\text { implant }\end{array}$ & & \multicolumn{2}{|c|}{497} & & \multicolumn{2}{|l|}{470} & \multicolumn{2}{|c|}{459} & & \\
\hline $\begin{array}{c}\text { Extraction }+ \\
\text { implant }+\mathrm{Na} \\
\text { hyaluronate }\end{array}$ & & \multicolumn{2}{|c|}{479} & & \multicolumn{2}{|l|}{468} & \multicolumn{2}{|l|}{472} & & \\
\hline
\end{tabular}

patients still had thickened corneas at six months after operation by a Graefe section with broad iridectomy. Recent series have shown a more rapid return to normal within six months to three weeks. ${ }^{56}$ In the present series there was return to normal limits within 28 days.

Sodium hyaluronate is a large polymer of repeating disaccharide units of $\mathrm{N}$-acetyl glucosamide and sodium glucosonate which is becoming increasingly used to protect the cornea during lens implantation and other intraocular procedures. Miller and Stegman ${ }^{7}$ using Binkhorst implants found that with sodium hyaluronate to reform the anterior chamber prior to lens implantation corneal thickness after surgery was significantly reduced. They also found that an $18 \%$ loss of endothelial cells compares well with that seen in non-implant eyes. Pope and Balazs, ${ }^{10}$ however, found no significant difference in corneal thickness if the sodium hyaluronate was used to reform the anterior chamber following cataract extraction, but there was a significant reduction in corneal thickness if it was used to protect the endothelium prior to cataract extraction. In this series the use of sodium hyaluronate following cataract delivery caused a significant reduction in corneal thickness when compared with the group not on sodium hyaluronate. This difference became statistically significant at the 84 -hour measurement.

Why the difference should be maximal at 84 hours is difficult to say. It may indicate the time period required for recovery of function of damaged rather than destroyed endothelial cells. The sodium hyaluronate may enhance endothelial cell recovery in some way, perhaps by diminishing the amount of prostaglandin released at the time of cell damage and so preventing further loss of cell function.

The clinical significance of increased corneal thickness in the medium term may not be very great, as in all cases the cornea returned to normal preoperative thickness within 28 days. However, changes in corneal thickness in the immediate postoperative period are an important indicator of surgical technique and corneal endothelial damage. Anything which helps to reduce endothelial damage in the short term must have some clinical benefit in the very long term.

\section{CONCLUSION}

Operative endothelial damage can be assessed by measuring corneal thickness. The maximum increase in thickness occurs within 24 hours. With modern implants, microsurgical techniques, and careful surgery no significant difference between plain intracapsular cataract extraction and intracapsular extraction plus lens implantation was found in this series. A statistically significant difference was shown in corneal thickness when patients on intracapsular lens extraction or lens implant with protection of the endothelium by means of sodium hyaluronate were compared with a group in which sodium hyaluronate was not used. 
I thank Mr W Rich, Mr J Thomsitt, the late Mr F C Schwerdt and Mr G Cantrell for allowing me to use their patients in this study. My thanks particularly to Mr W Rich for his encouragement and help in reading the manuscript, to $\mathrm{Mr} \mathrm{M}$ Hedgley for secretarial assistance, and the Photographic Department, Royal Devon and Exeter Hospital for the illustrations.

\section{References}

1 Pearce JL. Long term results of Binkhorst iris clip lens in senile cataract. Br J Ophthalmol 1972; 56: 319-21.

2 Miller D, Dohlmann CH. Effect of cataract surgery on the cornea. Trans Am Acad Ophthalmol Otolaryngol 1970; 74: $369-74$.

3 Norn MS. Pachometric study on the influence of corneal endothelial vital staining. Acta Ophthalmol (Kbh) 1973; 51: 679-86.
4 Wood JW, Maumanee AE. Corneal thickness after cataract surgery. Am J Ophthalmol 1975; 79: 631-4.

5 Reshmi CS, Kondrot EC. Pachymetric evaluation of corneal thickness after cataract extraction and intraocular lens implantation. J Am Intraocular Implant Soc 1976; 2: 17-20.

6 Cheung H, Sturrock GD, Rubinstein B, Bulpitt CJ. Endothelial cell loss and corncal thickness after intracapsular extraction and iris clip lens implantation: a randomised control trial (interim report). Br J Ophthalmol 1977; 61: 785-90.

7 Miller D, Stegman R. Use of sodium hyaluronate in humans. IOL Implantation. Am J Ophthalmol 1981; 13: 811-5.

8 Mishima S, Hedbys BO. Measurement of corneal thickness with the Haag-Streit pachometer. Arch Ophthalmol 1968; 80: 710-3.

9 Olsen T. Corneal thickness and endothelial damage after intracapsular cataract extraction. Acta Ophthalmol (Kbh) 1980; 58: 424-33.

10 Pope LG, Balazs EA. Use of sodium hyaluronate in human anterior segment surgery. Ophthalmology 1980; 87: 699-705. 